\title{
VARIATION IN ACTIVATION ENERGY AND NANOSCALE CHARACTERISTIC LENGTH AT THE GLASS TRANSITION
}

\author{
Ion Dranca \\ Chemistry Institute, Moldova Academy of Sciences \\ 3 Academiei Str., Chisinau MD2028, Moldova \\ drancai@yahoo.com; Tel.37322725490Fax.37322739854 \\ Current address: Department of Pharmaceutics, University of Minnesota, 55455, USA
}

\begin{abstract}
Differential scanning calorimetry has been used to study the $\alpha$-relaxation (glass transition) in virgin polystyrene (PS), PS-clay nanocomposite, amorphous indomethacin (IM), maltitol (Mt) and glucose (Gl). Variation of the effective activation energy $(E)$ throughout the glass transition has been determined by applying an advanced isoconversional method to DSC data on the glass transition. The relaxations have been characterized by determining the effective activation energies $(E)$ and evaluating the sizes of cooperatively rearranging regions at the glass transition $\left(V_{g}\right)$. The values of $V_{g}$ have been determined from the heat capacity data. The $\alpha$-relaxation demonstrates markedly larger values of $E\left(\sim 340 \mathrm{vs} \sim 270 \mathrm{~kJ} \mathrm{~mol}^{-1}\right)$ for the PS-clay system than for virgin PS. For IM in the glass transition region, the effective activation energy of relaxation decreases with increasing temperature from 320 to $160 \mathrm{~kJ} \mathrm{~mol}^{-1}$. In the $T_{\mathrm{g}}$ region $E$ decreases (from $\sim 250$ to $\sim 150 \mathrm{~kJ} \mathrm{~mol}^{-1}$ in maltitol and from 220 to $\sim 170 \mathrm{~kJ} \mathrm{~mol}^{-1}$ in glucose) with increasing $T$ as typically found for the $\alpha$-relaxation. It has been found that in the sub- $\mathrm{T}_{\mathrm{g}}$ region $E$ decreases with decreasing $T$ reaching the values $\sim 60$ (glucose) and $\sim 70$ (maltitol) $\mathrm{kJ}^{-1}$ that are comparable to the literature values of the activation energies for the $\beta$-relaxation. Heat capacity measurements have allowed for the evaluation of the cooperatively rearranging region in terms of the linear size The PS-clay system has also been found to have a significantly larger value of $V_{g}, 36.7 \mathrm{~nm}^{3}$ as compared to $20.9 \mathrm{~nm}^{3}$ for PS. Heat capacity measurements of IM have allowed for the evaluation of the cooperatively rearranging region (CRR) in term of linear size (3.4 $\mathrm{nm})$ and the number of molecules (90). The size of CRR have been determined as 3.1 (maltitol) and 3.3 (glucose) nm.
\end{abstract}

Keywords: activation energy; differential scanning calorimetry (DSC); $\alpha$ - and $\beta$-relaxation; advanced isoconversional method; kinetics (polym. and pharm.)

\section{Introduction}

Differential scanning calorimetry (DSC) is a major tool for measuring the glass transition or, so-called, $\alpha$-relaxations in polymer systems [1]. In particular, it has been successfully employed to explore glass transition in various nanostructured polymer systems, including thin films [2,3] and polymers confined to nanopores [4,5]. In DSC, the glass transition shows up as a heat capacity step. An increase in the heating rate $q$ causes this step to shift to a higher temperature. This effect is used in the popular method by Moynihan et al [6], for evaluating the effective activation energy, $E$, from the slope of the $q$ versus $T_{g}^{-1}$ plots.

The glass transition kinetics are commonly treated in the frameworks of the Tool [7]-Narayanaswamy [8]-Moynihan [9] (TNM) model that assumes the activation energy of the process to be constant. As fairly noted [10,11], the assumed Arrhenius temperature dependence disagrees with the typically observed Vogel [12]-Tamman [13]-Fulcher [14] (VTF) and/ or Williams-Landel-Ferry [15] (WLF) dependencies. In particular, Kovacs et al. [10], stressed that the applications of the TNM model should be limited "to systems in which viscosity obeys an Arrhenius dependence within and above the glass transition range". According to Angell [16], such systems are termed as strong glass-forming liquids, whereas the systems that obey the VTF/WLF behavior are called fragile liquids. The departure from the Arrhenius behavior is characterized by the dynamic fragility, $m$. In general, this parameter is the smallest for inorganic oxide glasses and the largest for polymeric glasses. Needles to say, the VTF/WLF behavior typically observed for polymers gives rise to an effective activation energy that varies with temperature [17]. In our previous communication [18], we proposed to use an isoconversional method to detect the variation in the activation energy throughout the glass transition. The application of the method was demonstrated for two polymers: polystyrene (PS) and poly(ethylene terephthalate) (PET). Why variation? Constant activation energy is usually the anticipated outcome of kinetic evaluations. This expectation appears to be supported by theoretical reasoning. The Arrhenius equation, eq (1),

$$
k=A \exp (-E / R T)
$$

where $E$ is the activation energy (the heat of activation), $A$ the preexponential factor, $T$ the temperature, $\mathrm{R}$ the gas constant, materializes Arrhenius hypothesis [19] that normal (i.e. inactive) molecules are in an endothermic equilibrium with active ones, which take part in the reaction. According to Arrhenius, $E$ is the heat absorbed in the process of 
transformation of inactive molecules into active ones, or, in other words, the heat (or energy) of activation. Owing to its thermodynamic meaning, $E$ was treated $[19,20]$ as a constant that is independent of the pathway taken by a system from an initial to a final state. Van't Hoff [20] expected only a minor temperature dependence of the activation energy as a result of the temperature dependence of the heat capacity.

Later, collision theory [21, 22] and activated-complex theory [23] introduced the idea of an energy barrier that has to be crossed over for a reaction to occur. From the standpoint of these theories, Arrhenius' activation is a parameter that is directly related to the height of the energy barrier. Unlike the Arrhenius theory, collision theory and activated complex treat the activation energy as a truly kinetic (dynamic) parameter, which is associated with reaction act itself. However, similar to Arrhenius theory, these theories treat the activation energy as an essentially constant parameter of a reaction system [24]. These theoretical considerations led to the formation of the concept of constant activation energy, which seems to be a very reasonable approximation for the gas phase where chemical transformations take place by a series of isolated binary collisions of molecules [25]. The temperature variation of the activation energy has alwys been a topic of considerable interest among kineticists. Hulett [26] published a short review, which appears to be the first attempt to systematize the effects causing deviations from the Arrhenius law. Although thermal analysis is not really concerned with the kinetics of gas phase reactions, it is worthy of note that even for these reactions the activation energy should show a temperature dependence because of the temperature dependence of the heat capacity of activation [26, 27].

There is a strong but rather unjustified tendency in the thermal analysis community to directly interpret the effective activation energy in terms of a free energy barrier. Also, there are many flawed methods (the Coats-Redfern method definitely being the champion in popularity) that use a single heating rate and directly deliver a single and constant value of the activation energy as well as a preexponential factor for any reactions, no matter how complex it might be [27]. By accepting the concept of variable activation energy as a practical compromise, people abandon the methods that invariably produce a single value of the activation energy and start using multiple run (isothermal and/ or nonisothermal) methods that allow for detecting reaction complexity [28-30]. Since this phenomenon is obviously inconsistent with the concept of constant activation energy, it seems reasonable to introduce a concept of variable activation energy. This is not only more practical, but also universal because it permits a constant activation energy as a special case of a variable activation energy [25].

PS and PS-clay nanocomposites. Implanting layered silicates into polymers is known [31] to modify dramatically various physical properties including thermal stability and fire resistance [32]. It has been found that compared to virgin PS the clay nanocomposites have a somewhat higher glass transition temperature [33,34] and mechanical modulus, [34, $35]$ decompose at significantly greater temperatures, $[33,36]$ and demonstrate a substantial decrease in the maximum heat release rate of on combustion [36-38].

It should be stressed that even when clay content is as lithe as $0.1 \%$, the initial decomposition temperature is increased by $40^{\circ} \mathrm{C}$ and the heat peak release rate is decreased by about $40 \%$ relative to virgin PS [36]. The mechanism of such remarkable effect is not yet well understood. Although the thermal behavior of polymer-clay nanocomposites has been studied extensively, the kinetic aspects of degradation and relaxation remain practically unknown. The importance of reliable kinetic analysis cannot be overestimated as it may provide information on the energy barriers of the process as well as offer mechanistic clues.

Indomethacin (IM). Obtaining crystalline pharmaceuticals in an amorphous form in certain cases leads to a significant increase in the bioavailability. A fundamental problem associated with solid amorphous drugs is that they

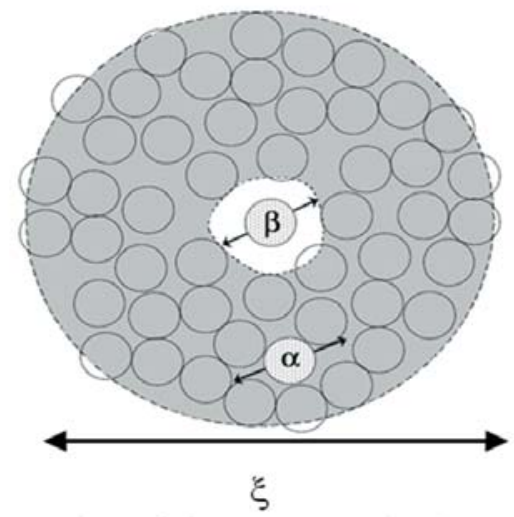

Fig 1. Schematic presentation of the cooperative $\alpha$-process and noncooperative $\beta$-process occurring in a cooperatively rearranging region of size $\xi$. The open area in the middle represents a mobility island 
are inherently unstable because of a strong thermodynamic drive toward crystallization. A great deal of studies has been concerned with evaluating the physical stability of amorphous drugs. Most of these studies have been focused on the dynamics of the glass transition ( $\alpha$-relaxation), because this is the process that on its heating transforms an amorphous system from a low- to high-mobility state, in which crystallization occurs very quickly. It is known that the molecular mobility associated with the $\alpha$-relaxation fades away rapidly as the temperature drops below the glass transition temperature, $T_{\mathrm{g}}$. Nevertheless, slow crystallization of indomethacin has been reported by several workers $[39,40]$ at temperature as low as $20^{\circ} \mathrm{C}$, which is about $25^{\circ} \mathrm{C}$ below the respective $T_{\mathrm{g}}$ value. On the other hand, relaxation in amorphous indomethacin is still detectable as much as $47{ }^{\circ} \mathrm{C}$ below $T_{\mathrm{g}}$ [41]. It seems to be generally thought thought that at temperature $50{ }^{\circ} \mathrm{C}$ below $T_{\mathrm{g}}$, the molecular mobility should be too negligible to cause any significant relaxation and, therefore, crystallization. This is certainly true for the $\alpha$-relaxation that requires a cooperative motion of multiple molecules and therefore has a huge energy to it. However, local noncooperative motion has a significantly smaller energy barrier and continues to occur at lower temperatures, giving rise to the $\beta$-process [46]. Although the $\beta$-relaxations (Fig 1) had been well known for polymers, Johari and Goldstein [42] discovered similar processes in simple inorganic glasses, and suggested these to be universal feature of the glassy state.

1.3 Glucose (Gl) and Maltitol (Mt). Sugar and sugar alcohols find a wide application in various pharmaceutical systems as sweeteners and/or binding matrices for drug components. As such they are frequently used in the glassy state, which is thermodynamically unstable and unavoidably tends to relax toward the liquid state [43]. Understanding the dynamics of this process is of a great practical importance for designing physically stable formulations of amorphous drugs.

In this work, we apply DSC to measure the $\alpha$-relaxation in a PS-clay system, in virgin PS, in amorphous indomethacin, and also in amorphous glucose and maltitol. The objective of this study is to obtain comparative information on the relaxation dynamics of these systems by estimating the effective activation energies of the relaxation as well as the sizes of cooperatively rearranging regions at the glass transition. This paper is intended to initiate systematic kinetic studies of polymer nanocomposites and pharmaceutic materials, therefore, to fill the presently existing void in the understanding of the thermal behavior of these exciting materials.

\section{Experimental Section}

A sample of the PS-clay composite was provided by Dr. Xiaowu Fan (Northwestern University). The composite was prepared by intercalating a monocationic free radical initiator into montmorillonite clay and the subsequent solution surface-initiated polymerization (SIP), where the chain growth was initiated in situ from clay surfaces. The montmorillonite clay was Cloisite ${ }^{\circledR} \mathrm{Na}^{+}$(Southern Clay Product Inc.) with the cation exchange capacity 92 mequiv per $100 \mathrm{~g}$ and the specific surface area $750 \mathrm{~m}^{2} \mathrm{~g}^{-1}$. The initiator was an azobisisobutyronitrile (AIBN)-analogue molecule with a quaternized amine group at one end. The intercalation process was realized by cation exchange reaction in which the cationic end of the initiator was ionically attached to the negatively charged clay surfaces. The details regarding the preparation and characterization of the intercalated clay can be found elsewhere [44]. The subsequent SIP was performed in THF solvent with styrene as the monomer, resulting in a PS-clay nanocomposite by the in situ polymerization. Details of the initiator synthesis, the SIP process and product analysis can be found in previous publication, which shows that this free radical SIP strategy can achieve exfoliated PS-clay nanocomposites of high clay loading. According to our pervious TGA [45] measurements the resulting composite contains about $1 \%$ of clay by weight. The molecular weight $(\sim 90,000)$ and polydispersity $(\sim 2.3)$ of the product were measured by size exclusion chromatography (SEC) using PS standards. The obtained material will be referred to as nPS90. For comparison purposes, we have used radically polymerized PS that was purchased from Alfa Aesar and used as received. Its Mw value is 100,000 (polydispersity is around 2.1) and it will be referred to as PS100.

The $\alpha$-relaxation measurements were performed on $\sim 10 \mathrm{mg}$ samples placed in $40 \mu \mathrm{L} \mathrm{Al}$ pans. The glass transition was measured by heating a sample $\sim 40^{\circ} \mathrm{C}$ above its glass transition temperature and holding it at this temperature for 10 min to erase thermal history. The samples were then cooled down to $\sim 40^{\circ} \mathrm{C}$ below the glass transition temperature at the rates form $5,7.5,10,12.5,15,17.5,20,22.5$ and $25^{\circ} \mathrm{C} \mathrm{min}{ }^{-1}$. Immediately after completion of the cooling segment, the samples were heated at a rate whose absolute value was equal to the rate of preceding cooling. TGA data [45] indicate that no detectable degradation of PS100 and nPS90 occurs in air below $240^{\circ} \mathrm{C}$.

Indomethacin (1-(p-chlorobenzoil)-5-methoxy-2-methylindole-3-acetic acid) was obtained from MPBiomedicals, LLC (catalogue number 190217, lot number 9331E), with a melting point at $160 \mathrm{oC}$ obtained by DSC, and it was used without further purification.

The $\alpha$-relaxation measurements were performed on $\sim 10.0 \mathrm{mg}$ samples placed in $40 \mu \mathrm{L} \mathrm{Al}$ sealed pans without holes. TGA data [46] indicate that no detectable degradation of Indomethacin occurs in air atmosphere below $180^{\circ} \mathrm{C}$. The glass transition was measured by heating a sample $\sim 40^{\circ} \mathrm{C}$ above its glass transition temperature $\left(90^{\circ} \mathrm{C}\right)$ and holding it at this temperature for $10 \mathrm{~min}$ to erase thermal history. The samples were then cooled down to $\sim 40^{\circ} \mathrm{C}$ below the glass transition temperature $\left(-10^{\circ} \mathrm{C}\right)$ at the rates form $\left(10,15,20,25\right.$ and $\left.30^{\circ} \mathrm{C} \mathrm{min}{ }^{-1}\right)$. Immediately after completion of the 
cooling segment, the samples were heated at a rate whose absolute value was equal to the rate of preceding cooling. The heat capacity was measured on $\sim 27 \mathrm{mg}$ sample by using a standard procedure that is precise to $1 \%$. A sapphire sample of $41.48 \mathrm{mg}$ was used as the calibrant. The temperature program used for the heat capacity measurement involved 5 min isothermal hold at $0^{\circ} \mathrm{C}$ followed by heating at $10^{\circ} \mathrm{C} \mathrm{min}^{-1}$ to $90^{\circ} \mathrm{C}$ and another 5 min isothermal hold at the final temperature.

Anhydrous glucose (Dextrose) and maltitol were, respectively, purchased from Fisher and MP Biomedicals and used without purification. In order to produce amorphous (glassy) samples, $\sim 15 \mathrm{mg}$ of samples was placed in 40 $\mu \mathrm{l}$ closed $\mathrm{Al}$ pans and heated to $\sim 10 \mathrm{oC}$ above their respective melting points, $161^{\circ} \mathrm{C}$ (glucose) and $149^{\circ} \mathrm{C}$ (maltitol). Shortly after heating the samples were quenched into liquid nitrogen. The glass transition temperatures of amorphous samples were estimated as midpoint temperatures of the DSC glass transition steps measured at $10^{\circ} \mathrm{C} \min ^{-1}$.

Sub- $T_{g}$ measurements, freshly quenched samples were quickly placed into the DSC cell that was maintained at $-40^{\circ} \mathrm{C}$. From $-40^{\circ} \mathrm{C}$ the samples were heated to an annealing temperature, $T_{\mathrm{a}}$, and held at it for $30 \mathrm{~min}$. After completion of the annealing segment, the samples were cooled down to $-40^{\circ} \mathrm{C}$ and immediately heated above $T_{\mathrm{g}}$. The heating rates

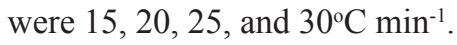

All the aforementioned measurements we conducted by using a Mettler-Toledo heat flux DSC 822e in the atmosphere of nitrogen flow $\left(80 \mathrm{ml} \mathrm{min}^{-1}\right)$ at University of Alabama, USA. The temperature and heat flow calibration were performed by using an Indium standard.

\section{Results and Discussion}

Glass transition or $\alpha$-relaxation in PS100 and nPS90. DSC data for both materials demonstrate (Fig. 1) typical glass transition steps, the midpoint of which has been used as an estimate of the $T_{g}$ value. It is seen that in nPS90 the glass transition occurs at about 10 oC greater temperature than PS100.

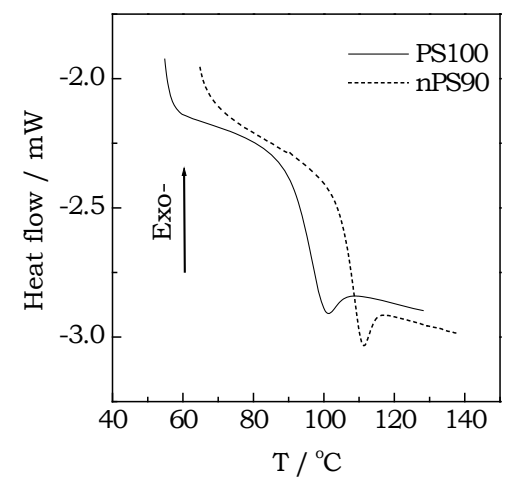

Fig. 2. DSC curves for the glass transition ( $\alpha$-relaxation) in PS100 and nPS90 measured at $10{ }^{\circ} \mathrm{Cmin}^{-1}$

The values of $T_{g}$ have been determined at nine heating rates (two times at each heating rate). The heating rate dependence of $\mathrm{Tg}$ can be used to evaluate the effective activation energy of the glass transition from eq 1.

$$
E=-R \frac{d \ln \beta}{d T_{g}}
$$

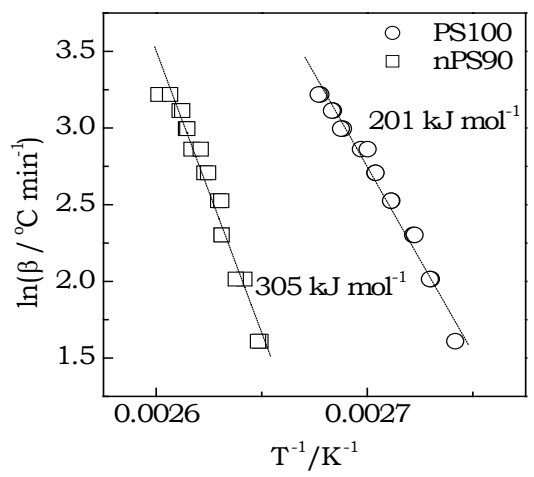

Fig. 3. Evaluating activation energies (eq 2) for $\alpha$-relaxation in PS100 and nPS90 as proposed by Moynihan et al. [6, 47] 
A necessary constraint of eq 2 , is that the sample must be heated at a rate whose absolute value is equal to the rate of preceding cooling $[6,47]$. The resulting dependence of $\ln \beta$ versus $T_{g}^{-1}$ are shown in Fig. 3. The plots are nonlinear, which is typically observed for the $\alpha$-relaxations $[11,48]$. The effective activation energy decreases with temperature, which is consistent with the general tendency predicted by the WLF equation $[15,17]$ :

$$
E=2.303 R \frac{c_{1} c_{2} T^{2}}{\left(c_{2}+T-T_{g}\right)^{2}}
$$

where $c_{1}$ and $c_{2}$ are the constants. Forcing data to an Arhenius plot yields averaged values of the effective activation energy for PS100 and nPS90 that respectively are 201 and $305 \mathrm{~kJ} \mathrm{~mol}^{-1}$. To determine a variation of $E$ with the extent of conversion from the glassy to rubbery state, $\alpha$, we have used an advanced isoconversional method [49, 50]. The method offers two major advantages over the frequently used methods of Flyn and Wall [51] and Ozawa [52]. First, it has been designed to treat the kinetics that occur under arbitrary variation in temperature, $T(t)$, which allows one to account for self-heating/cooling detectable by the thermal sensor of the instrument. For a series of $n$ experiments carried out under different temperature programs, $T(t)$, the activation energy is determined at any particular value of $\alpha$ by finding $E_{\alpha}$, which minimizes the function

$$
\Phi\left(E_{\alpha}\right)=\sum_{i=1}^{n} \sum_{j \neq 1}^{n} \frac{J\left[E_{\alpha}, T_{i}\left(t_{\alpha}\right)\right]}{J\left[E_{\alpha}, T_{j}\left(t_{\alpha}\right)\right]}
$$

where

$$
J\left[E_{\alpha}, T_{i}\left(t_{\alpha}\right) \equiv \int_{t_{\alpha}-\Delta \alpha}^{t_{\alpha}} \exp \left[\frac{-E_{\alpha}}{R T_{i}(t)}\right] d t\right.
$$

The second advantage is associated with performing integration over small time segments (eq 4), which allows for eliminating a systematic error [50] occurring in the Flynn and Wall and Ozawa and Ozawa methods when $E_{\alpha}$ varies significantly with $\alpha$. In eq $5, \alpha$ is varied from $\Delta \alpha$ to $1-\Delta \alpha$ with a step $\Delta \alpha=m^{-1}$, where $m$ is the number of intervals chosen for analysis. The integral, $J$ in eq 4 , is evaluated numerically by using the trapezoid rule. The minimization procedure is repeated for each value of $\alpha$ to find to find the dependence $E_{\alpha}$ on $\alpha$. Isoconversional methods determine the $E_{\alpha}$ values independently of the preexponential factors, which a not produced directly by these methods. It allows one to eliminate the bias in the value of the activation energy caused by its strong correlation with the exponential factor that is generally found when both parameters are fit simultaneously [53]. The conversion, $\alpha$, can be evaluated from DSC data (Fig. 2) as the normalaized heat capacity [54] as follows:

$$
C_{p}^{N}=\frac{\left.\left.\left(C_{p}-C_{p g}\right)\right|_{p g}\right)\left.\right|_{T}}{\left(C_{p l}-C_{p}\right.} \equiv \alpha
$$

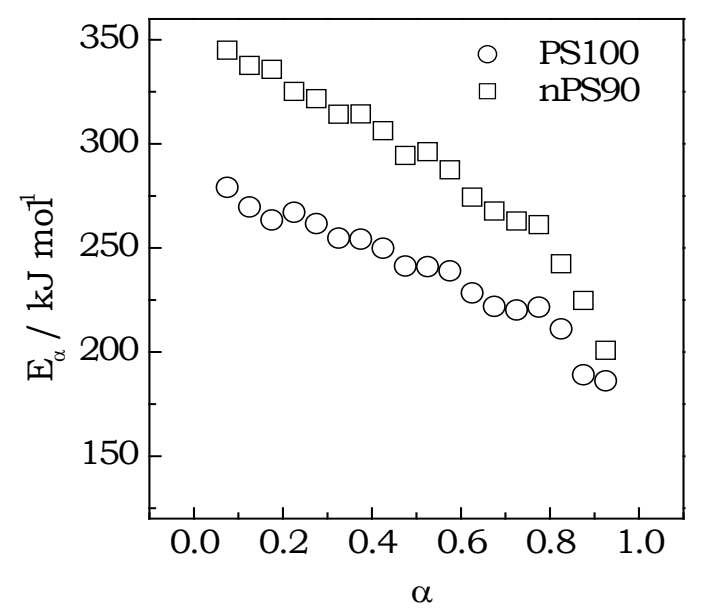

Fig. 4. Variation of the activation energy for $\alpha$-relaxation with the extent of conversion 
where $C_{p}$ is the observed heat capacity, and $C_{p g}$ and $C_{p l}$ are respectively the glassy and equilibrium (liquid) heat capacity. Because the values $C_{p g}$ and $C_{p l}$ are temperature dependent, they must be extrapoled into the glass transition region.

According to Hodge, [54] the $C_{p}{ }^{N}$ value provides a precise a precise approximation to the temperature derivative of the fictive temperature. The application of the isoconversional method to the resulting $\alpha$ versus $T$ data obtained at different heating rates yields the $E_{\alpha}$ dependencies shown in Figure 4. The activation energies decrease from 280 to 140 $\mathrm{kJ} \mathrm{mol}^{-1}$ for PS100 and from 350 to $160 \mathrm{~kJ} \mathrm{~mol}^{-1}$ for nPS90. Note that the values obtained for PS100 fall within the large interval, 160-900 $\mathrm{kJ} \mathrm{mol}^{-1}$, reported in the literature [54-59].

Volume of Cooperatively Rearranging Regions in PS100 and nPS90. On the basis of the result discussed in the previous section, the polymer-clay system should have a larger region of cooperative rearrangement. Donth has demonstrated that the volume of the cooperatively rearranging region at the glass transition can be estimated from calorimetric data by using the following equation $[48,60]$ :

$$
V_{g}=\frac{k_{B} T_{g}^{2} \Delta\left(\begin{array}{l}
-1 \\
v
\end{array}\right)}{\rho(\delta T)^{2}}
$$

$k_{B}$ is the Boltzman constant, $T_{g}$ is an apparent glass transition temperature, $\rho$ is the density $\left(1.05 \mathrm{~g} \mathrm{~cm}^{-3}\right.$ for PS [61]), and $C_{v}$ is the isohoric heat capacity. The value of $\Delta\left(C_{v}^{-1}\right)$ is determined as

$$
\Delta\left(C_{v}^{-1}\right)=\left(C_{v g}^{-1}-C_{v l}^{-1}\right.
$$

where $C_{v g}$ and $C_{v l}$ are respective values of the glassy and liquid heat capacity extrapoled to $T_{g}$. The difference between isohoric and isobaric heat capacities is usually neglected [60] so that $C_{v}$ can be replaced with $C_{p}$ which is readily obtainable from calorimetric measurements. More recently, Hempel et al. [62] demonstrated that the difference can be accounted via the following correction:

$$
\Delta\left(C_{v}^{-1}\right)=(0.74 \pm 0.22) \Delta\left(C_{p}^{-1}\right)
$$

If the glass transition is measured on heating, the mean temperature fluctuation, $\delta T=\frac{\Delta T}{2.5}$

where $\Delta T$ is the temperature interval within which $C_{p}$ varies between 16 and $84 \%$ of the total $\Delta C_{p}$ at $T_{g}$ [60 62].

Figure 5, shows the results of our $C_{p}$ measurements for PS100 and nPS90.

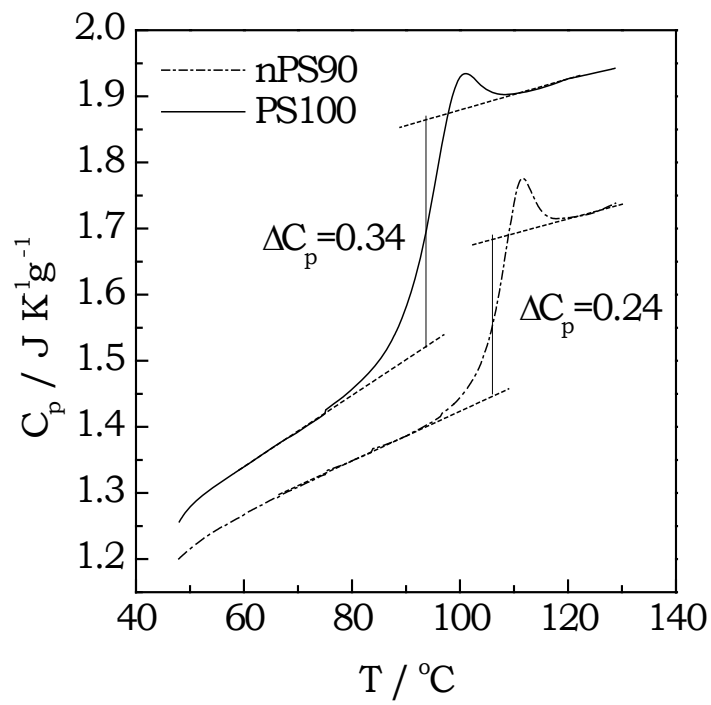

Fig. 5. Temperature dependence of the heat capacity for PS100 and nPS90

The values of $C_{p}$ for PS are in very good agreement with earlier measurements [63, 64]. 
Size of Cooperatively Rearranging Region and Experimental Parameters Used for Its Calculations

\begin{tabular}{|l|c|c|c|c|c|}
\hline sample & $T_{g}, \mathrm{~K}$ & $C_{p g}, \mathrm{~J} \mathrm{~K}^{-1} \mathrm{~g}^{-1}$ & $C_{p l}, \mathrm{~J} \mathrm{~K}^{-1} \mathrm{~g}^{-1}$ & $\delta T, \mathrm{~K}$ & $V_{g}, \mathrm{~nm}^{3}$ \\
\hline PS100 & 366.9 & 1.53 & 1.87 & 2.6 & 20.9 \\
\hline nPS90 & 379.4 & 1.45 & 1.69 & 1.9 & 36.7 \\
\hline
\end{tabular}

It is seen that the $C_{p}$ values for the PS-clay system are noticeably smaller than for virgin PS. The decrease cannot be explained by the simply presence of clay because it is present in a very small amount (1\%) and its heat capacity [65] is comparable to that of PS. On the other hand, the value of heat capacity is proportional to the number of internal degrees of freedom of molecular motion. By applying 7-10 to the heat capacity data (Fig. 5), we have evaluated the volume of cooperatively rearranging region PS100 and nPS90 (Table 1). The values $\zeta=\left(V_{g}\right)^{1 / 3}$ gives the characteristic length of the cooperatively rearranging region $[48,60]$. According to our data for PS100 (Table 1), $\xi$ is $2.8 \mathrm{~nm}$, which agrees well with the value $3.0 \mathrm{~nm}$ recently reported by Hempel et al. [62].

Glass transition or $\alpha$-relaxation in Indomethacin. By applying the isoconversional method $[49,50]$ to the $\alpha$ versus $T$ data for different heating rates, [46] we obtained the $E_{\alpha}$ dependence shown in Fig. 6.

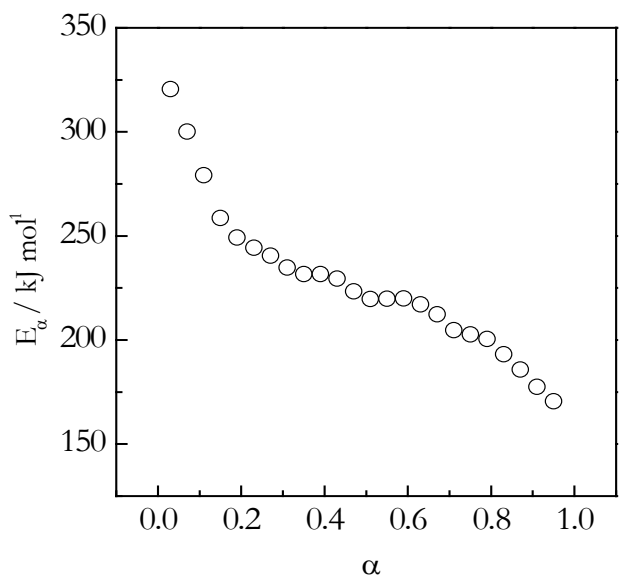

Fig 6. Variation of the activation energy for the $\alpha$-relaxation with the extent of relaxation for the glass transition in indomethacin

The effective activation energy decreases from 320 to $160 \mathrm{~kJ} \mathrm{~mol}^{-1}$. A decrease in $E$ with the transition from the glassy to liquid state has also been reported by Hancock et al. [66], who estimated $E$ for the onset, midpoint, and offset of the transition, and obtained respective values of 385,263 , and $190 \mathrm{~kJ} \mathrm{~mol}^{-1}$

The decrease in $E$ can be explained in terms of cooperative molecular motion. The glassy state has a small amount of free volume that permits only the local motion (i.e., the $\beta$-process) (Fig. 1) that persists well below the glass transition temperature. As the temperature increases and approaches the region of the glass transition, the molecular motion becomes more intense, and the free volume rises, initiating the $\alpha$-process. This process needs a great degree of cooperativity between the molecules, which gives rise to a large energy barrier represented by a large value of $E$ at the initial stage of transition. As the free volume grows with increasing temperature, the molecular packing becomes looser, allowing molecules to relax more independently (i.e., with a smaller degree of cooperativity). As a result, the energetic constraints relax, and the effective activation energy drops. Note that a fall of $E$ is consistent with both the WLF and VTF equations [12-15]. A decrease is also predicted by the Adam-Gibs equation [67]

$$
\tau=A \exp \left(\frac{z^{*} \Delta \mu}{k_{B} T}\right)
$$


where $k_{B}$ is the Boltzman constant, $\Delta \mu$ is the activation energy per particle, and $z^{*}$ is the number of particles that rearrange cooperatively. In eq $11, z^{*}$ is inversely proportional to the configurational entropy that rises with $T$, so that both $z^{*}$ and the effective activation energy (i.e., $z^{*} \Delta \mu$ ) decrease with increasing $T$.

Size of Cooperatively Rearranging Regions in Indomethacin. Figure 7 shows a $C_{\mathrm{p}}$ versus $T$ curve that is an average of three independent measurements.

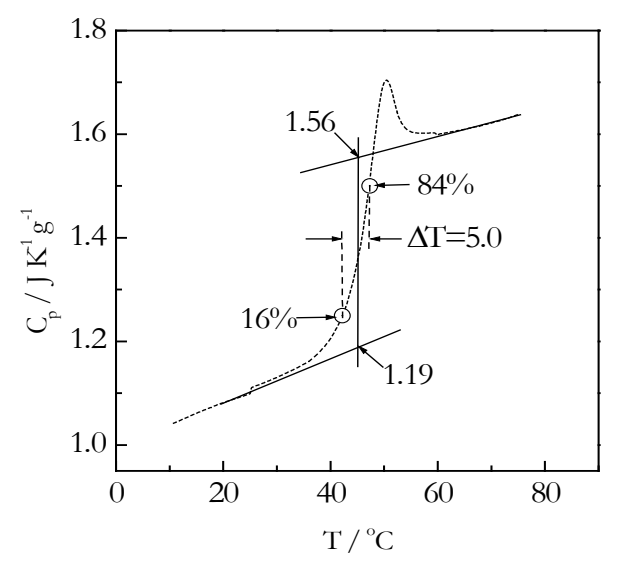

Fig. 7. Temperature dependence of the heat capacity for indomethacin. The heat capacity at the glass transition $(319 \mathrm{~K})$ changes from 1.19 to $1.56 \mathrm{~K}^{-1} \mathrm{~g}^{-1} . \Delta T$ is determined as the temperature interval in which $C_{p}$ changes from 16 to $84 \%$ of the total $\Delta C_{p}$.

The obtained $C_{\mathrm{p}}$ data agree very well with the earlier measurements [68]. The application of eqs 7-10 to the $C_{\mathrm{p}}$ data (Figure 6) yields the volume of the cooperatively rearranging region of $40.5 \mathrm{~nm}^{3}$. The characteristic length of the cooperatively rearranging region (Figure 1) or the average distance between the mobility islands [42] estimated as $\xi=\left(V_{\alpha}\right)^{1 / 3}$ is $3.4 \mathrm{~nm}$. The value is comparable to that estimated for sorbitol, $3.6 \mathrm{~nm}$ [62] The numbers of molecules involved in the cooperatively rearranging region is determined as

$$
N_{\alpha}=\frac{R T_{g}^{2} \Delta\left(C_{v}^{-1}\right)}{M(\delta T)^{2}}
$$

where $R$ is the gas constant and $M=357.8 \mathrm{~g} \mathrm{~mol}^{-1}$ is the molecular weight of indomethacin. The resulting value is $\sim 90$ molecules.

Glass transition or $\alpha$-relaxation in Glucose and Maltitol. Heating glassy maltitol and glucose throughout the temperature region of the glass transition results in obtaining typical step-like DSC traces (Fig.8).

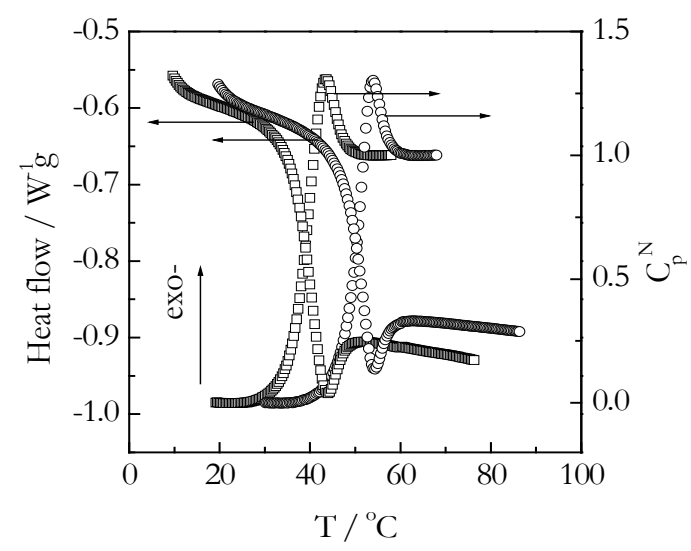

Fig 8. DSC curves and normalized heat capacities for the glass transition of maltitol (circles)

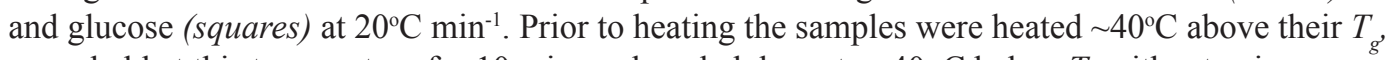
held at this temperature for $10 \mathrm{~min}$, and cooled down to $\sim 40 \mathrm{oC}$ below $T_{g}$ without aging. 
The steps shift to higher temperature with increasing the heating rate. The application of the isoconversional method to several systems demonstrated [18] that the variability in $E$ correlates with the dynamic fragility. For maltitol and glucose the reported values of the dynamic fragilitry, respectively, are 75 [69] and 70 [70]. As these values are not very large, we may expect a moderate variability in $E$ throughout the glass transition. The actual dependencies of the effective activation energy on conversion are shown in Fig. 10. As expected, we observe a moderate decrease for both systems. For maltitol the activation energy decreases from $\sim 250$ to $\sim 150 \mathrm{~kJ} \mathrm{~mol}^{-1}$ whereas for glucose from $\sim 220$ to $\sim 170 \mathrm{~kJ} \mathrm{~mol}^{-1}$.

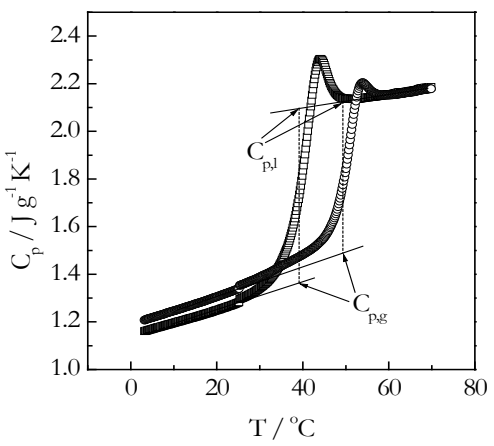

Fig. 9. Temperature dependence of the heat capacity for maltitol (circles) and glucose (squares).

$C_{p \cdot r}$ and $C_{\mathrm{p} \cdot \mathrm{g} .}$ show the values extrapoled to the $T_{g}$ values (dashed lines) that are used in evaluating $\xi$ by eq. 7 .

The $E_{\alpha} v s \alpha$ plots (Fig. 10) were converted into $E_{\alpha} v s T$ plots by replacing $\alpha$ with temperature which is estimated as an average of the temperatures corresponding to this $\alpha$ at different heating rates.

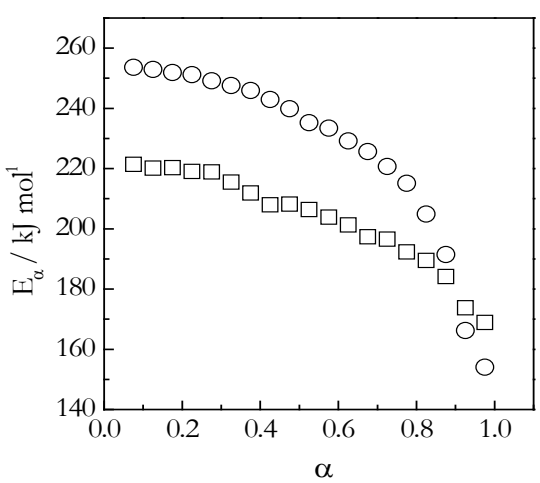

Fig. 10. Variation of the activation energy with the extent of relaxation for the glass transition in maltitol (circles) and glucose (squares).

The resulting dependencies are shown in Fig.11 and 12. As mentioned earlier, a decrease in the effective activation energy with temperature is typical for the glass transition ( $\alpha$-relaxation). We observed similar effects for polystyrene and polystyrene composite [71] as well as for poly(ethylene terephthalate) and boron oxide [18]. For the most fragile system studied [18], poly(ethylene terephthalate) $(m=166)$, the value of $E$ decreased more than 2 times per $7^{\circ} \mathrm{C}$, whereas for boron oxide $(m=32)$ it decreased less than 1.5 times per $57^{\circ} \mathrm{C}$.

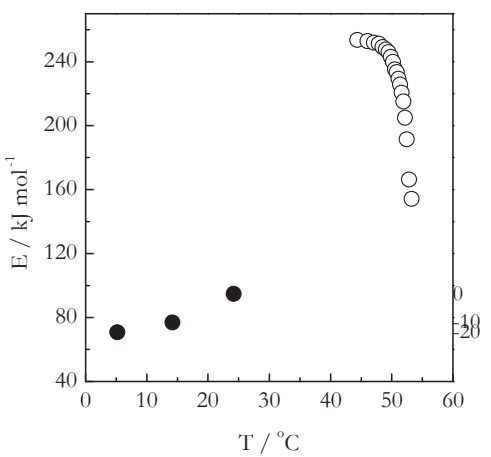

Fig.11. Variation of the activation energies for the sub- $T_{\mathrm{g}}$ (solyd symbols) and $T_{\mathrm{g}}$ (open symbols) relaxation of maltitol with average temperature of the process. Numbers by the points represent annealing temperatures. 
Maltitol and glucose have intermediate fragilities of about the same value so that they demonstrate similar variability in $E$ that amounts to 1.7 times $10^{\circ} \mathrm{C}$ for maltitol and 1.3 times per $8^{\circ} \mathrm{C}$ for glucose. Therefore, the correlation of the variability in $E$ with the fragility appear to hold for these two systems as well.

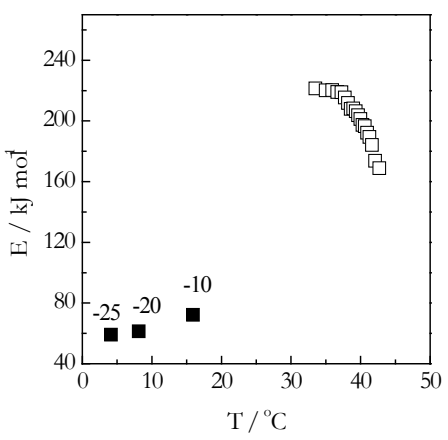

Fig. 12. Variation of the activation energies for the sub- $T_{\mathrm{g}}$ (solid symbols) and $T_{\mathrm{g}}$ (open symbols) relaxation of glucose with average temperature of process. Numbers by the points represent annealing temperatures.

The Arrhenius activation energies reported for the $\alpha$-relaxation in maltitol and in glucose span a rather wide range which is not surprising as their values depend on the temperature and, therefore, on the temperature region used their determination. For this reason, single value of $E\left(440\right.$ [72] and 460 [69] kJ mol$\left.{ }^{-1}\right)$ reported for maltitol are difficult to compare with our variable values. On the other hand, our dependence fits well within the reported [73] decrease in $E$ from 560 to $50 \mathrm{~kJ} \mathrm{~mol}^{-1}$ with increasing temperature. Single values of $E$ for the $\alpha$-relaxation in glucose are 180, [74] 320 [75], and $420 \mathrm{~kJ} \mathrm{~mol}^{-1}$ [70].

It should be stressed that the effective activation energy landscapes presented in Figs. 11 and 12 are remarkably similar to the temperature dependence of the effective activation energy for stress relaxation as well as for viscous flow in polymers. These latter also show a significant increase in $E$ on approaching the glass transition region from the glassy state, followed by a significant decrease in $E$ while further relaxing toward the liquid state. This effect was originally predicted by Fox and Flory [76] and observed experimentally by McLoughlin and Tobolsky [77]. Passing of the $E$ value through a maximum can be understood in terme of molecular cooperativity that is very low significantly below $T_{\mathrm{g}}$ where relaxation occurs via a noncooperative local process that has a small activation energy. As the temperature rises, the molecular mobility intensifies increasing cooperativity and the activation energy that reach their maximum in the region of $T_{\mathrm{g}}$. Above $T_{\mathrm{g}}$, the free volume starts to quickly increase with temperature so that cooperativity decreases, and the effective activation energy drops down to the values characteristic of the viscous flow.

3.5. Sizes of Cooperatively Rearranging Regions in maltitol and glucose. Figure 9 displays $C_{\mathrm{p}} v s$ data for maltitol and glucose. The data agree well with the earlier measurements $[69,78]$. By applying Eqs. 7, 8, 9, 10 to the $C_{\mathrm{p}}$ data (Fig. 9) we determined the values of $V_{\alpha}$ to be 30.6 (maltitol) and 36.4 (glucose) $\mathrm{nm}^{3}$. The values of the characteristic length were determined as $\xi=\left(V_{\alpha}\right)^{1 / 3}$ yelding, respectively, 3.1 and $3.3 \mathrm{~nm}$. The values are very close to each other and comparable to the value reported [62] for sorbitol, $3.6 \mathrm{~nm}$. By its meaning the value of $\xi$ provides an estimate of the average distance between the mobility islands in the heterogeneous glassy system [48]. The closeness of the $\xi$ values suggests similarity of the heterogeneous structures of the maltitol and glucose glasses. The $C_{\mathrm{p}}$ data can also be used to estimate the number of molecules involved in the cooperatively rearranging region as [48].

The resulting values are $\sim 90$ for maltitol and 190 for glucose. The value for glucose is almost the same as for sorbitol ( $N_{\alpha}=195$ molecules [48]), whose molecular mass is similar to that of glucose.

3.6. Sub- $T_{\mathrm{g}}$ Region. While reheating polyvinyl chloride samples annealed significantly below the glass transition temperature, $T_{\mathrm{g}}$, Illers [79], detected small endothermic DSC peaks that appears before the main glass transition step. The effect was later studied more extensively for metallic glasses by Chen [80] and for various polymers by Bershtein and Egorov [81]. An interpretation of the effect was given by Chen as a partial enthalpy relaxation and recovery that occur at the expense of the faster part of a broad relaxation spectrum of the glassy state. It was suggested [80, 81] that effective activation energy, $E$ of the underlying process can be determined from the shift in the annealing peak temperature, $T_{\mathrm{p}}$ with the heating rate, $q$ as

$$
E=-R \frac{\ln q}{d T_{p}^{-1}}
$$

where $R$ is the gas constant. The $E$ values determined this way were found [80,81] were found to be several times smaller than the activation energies of the $\alpha$-relaxations in the respective glassy systems. The annealing peaks are 
easily produced by annealing a glassy material in the region around $0.8 T_{\mathrm{g}}$. It has been pointed that the dielectric and mechanical loss peaks for $\beta$-relaxation in polymers are commonly found at temperature around $0.75 T_{\sigma}$ [82]. For maltitol, we were able to obtain good annealing peaks at $T_{\mathrm{a}}=-20,-10$, and $0^{\circ} \mathrm{C}$. A representative example of the annealing peaks obtained for maltitol is shown in Fig. 13.

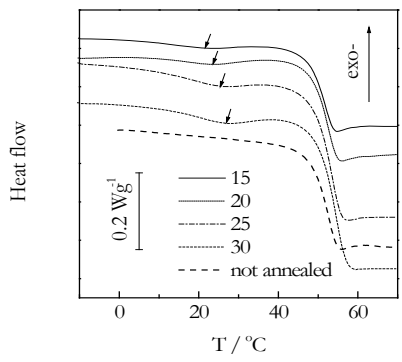

Fig. 13. DSC curves obtained on heating of maltitol at the heating rates $15-30^{\circ} \mathrm{C} \mathrm{min}^{-1}$

(numbers by the lines represent the heating rates) after annealing at $0^{\circ} \mathrm{C}$ for $30 \mathrm{~min}$. "Not annealed"

curve obtained by heating a sample immediately after quenching. Arrows show the location of the annealing effect.

The resulting peaks are very broad and shallow but readily noticeable especially when comparing DSC for annealed samples against those for not annealed samples. At a constant annealing temperature, the peak temperature increase with increasing the heating rate (Fig. 13) that allowed us to estimate the effective activation energies by Eq.13. The respective $\ln q v s T^{1}$ plots are shown in Fig. 14. It is seen the slopes of the plots increase with increasing the annealing temperature. This means that the effective value of $E$ rises with $T_{a}$. For maltitol, the obtained values of $E$ are 71,77 , and $95 \mathrm{~kJ} \mathrm{~mol}^{-1}$ (Fig. 11), for glucose, $E$ rises from 59 to 61 and to $72 \mathrm{~kJ} \mathrm{~mol}^{-1}$ (Fig. 12). The values are obviously smaller than the activation energies of the $\alpha$-relaxation energies whose typical values for sugars lie in the region 200-400 $\mathrm{kJ} \mathrm{mol}^{-1}$. The smaller values of $E$ sugest that the underlying relaxation process is either noncooperative or weakly cooperative (Fig. 1). Apparently, the observed increasing dependence of $E$ with $T_{\mathrm{a}}$ reflects an increasing contribuition of the cooperative molecular motion that stars to unfreeze as the annealing temperature approaches to the glass transition, $\left(T_{\mathrm{q}}\right)$ region. For the $\beta$-relaxation in maltitol the reported values of the activation energy are as follow: 57 [72] and 61 [73] by using dielectric spectroscopy. For glucose, our best estimate is $59 \mathrm{~kJ} \mathrm{~mol}^{-1}$. The literature reports the following values for the activation energy of the $\beta$-relaxation: 42 [75], 52 [83], and 62 [84] kJ mol ${ }^{-1}$, all of which were determined by using dielectric spectroscopy. Clearly, our estimate falls within the region of the reported values that constitutes its good correlation with activation energies of the $\beta$-relaxation.

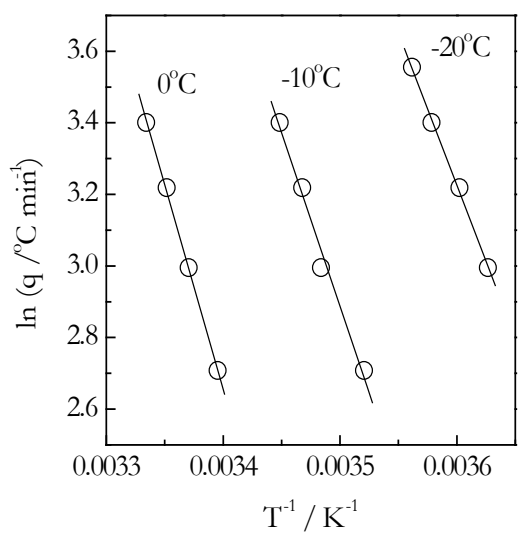

Fig. 14. Evaluating activation energies (Eq. 13) for sub- $T_{\mathrm{g}}$ relaxation of maltitol annealed at different temperatures. The magnitudes of the annealing temperatures shown by the straight lines.

Lastly, both estimates for maltitol and glucose, fit well into the empirical correlation, $E_{\beta}=(24 \pm 3) R T_{\mathrm{g}}$ reported by Kudilik et al. [85. According to this, the activation energies of the $\beta$-relaxation in maltitol and glucose, should respectively, lie within $\sim 8 \mathrm{~kJ} \mathrm{~mol}^{-1}$ limits of 64 and $62 \mathrm{~kJ} \mathrm{~mol}^{-1}$.

\section{Conclusions}

DSC can be used to determine the activation energies of the $\alpha$ - and $\beta$-relaxations and for evaluating the sizes of cooperatively rearranging regions at the glass transition in polymer-clay systems and pharmaceutics. 
A dramatic difference from virgin PS and PS-clay system has been observed for $\alpha$-relaxation, which demonstrates a significant increase in both effective activation energy and size of cooperatively rearranging region for the PS-clay system. Both values suggest that transitional motion in the PS-clay system require extra cooperativity.

For indomethacin, in the glass transition region, the effective activation energy of relaxation decreases with increasing temperature from 320 to $160 \mathrm{~kJ} \mathrm{~mol}^{-1}$.

The sub- $T_{\mathrm{g}}$ relaxation data for maltitol and glucose suggest that with decreasing the annealing temperatures the effective activation energies decrease and tend to converge to the activation energies of the $\beta$-relaxation. In sub- $T_{\mathrm{g}}$ and $T_{\mathrm{g}}$ regions the effective activation energies for maltitol are somewhat greater than for glucose that appears to be associated with the added impeding effect of the bulky substitute group in maltitol.

The comparable sizes of the cooperatively rearranging regions suggest a similarity of the heterogeneous glassy structures of the two compounds.

Therefore, DSC can be effectively used to determine the activation energy landscape for relaxation of nonpolymer glasses in a wide temperature range covering both the $\alpha$ - and $\beta$-relaxation. The resulting information on the effective activation energies and respective temperatures is of a great practical importance for evaluating the physical stability of amorphous polymers and pharmaceuticals. It allows one to detect the mobility and identify its type (cooperative $v s$ noncooperative) in a given temperature region.

Acknowledgment. Thanks are due to Mettler-Toledo, Inc. for loan of the DSC and TGA instruments used in this work.

\section{References}

[1] Wunderlich, B. Thermal Analysis; Academic Press: Boston, 1990.

[2] Bernazzani, P.; Simon, S. L.; Plazek, D. J.; Ngai, K. L. Eur. Phys. J. E. 2002, 8, 201.

[3] Efremov, M. Y.; Olson, E.A.; Zhang, M.; Zhang, Z.; Allen, L. H. Phys. Rev. Lett. 2003, 91, 085703.

[4] Park, J. Y.; McKenn, G. B.; Phys. Rev. B 2000, 61, 6667.

[5] Schonhals, A.; Goering, H.; Schick, C.; Frick, B.; Zorn, R. Eur. Phys. J. E, 2003, 12, 173.

[6] Moynihan, C.T.; Eastel, A.J.; Wilder, J.; Tucker, J. J. Phys. Chem. 1974, 78, 2673.

[7] Tool, A. Q. J. Am. Ceram. Soc. 1946, 29, 240.

[8] Narayanaswamy, O. S. J. Am. Ceram. Soc. 1971, 54, 491.

[9] Moynihan, C.T.; Eastel, A.J.; DeBolt, M. A.; Tucker, J. J. Am. Ceram. Soc. 1976, 59, 12.

[10] Kovacs, A. J.; Hutchinson, J.M.; Aklonis, J.J. The Structure of Non-Crystalline Materials; Gaskell, P.H. Ed: Taylor \& Francis, 1977, p. 153.

[11] Matsuoka, S. Relaxation Phenomena in Polymers; Hanser Publishers: Munich, 1992.

[12] Vogel, H. Phys. Z. 1921, 22, 645.

[13] Tamman,G.; Hesse, G. Z. Anorg. Allg. Chem. 1926, 156, 245.

[14] Fulcher, G. S. J. Am. Ceram. Soc. 1925, 8, 339.

[15] Williams, M. L.; Landel, R. F.; J. D. J. Am. Chem. Soc. 1955, 77, 3701.

[16] Angell, C. A. J. Non-Cryst. Solids 1991, 13, 131.

[17] Ferry, J. D. Viscoelastic Properties of Polymers, $3^{\text {rd }}$ ed.; J. Wiley: New York, 1980.

[18] Vyazovkin, S.; Sbirrazzuoli, N.; Dranca, I. Macromol. Rapid Commun. 2004, 25, 1708.

[19] Arrhenius, A. Phys. Chem., 1889, 4, 226.

[20] Van’t Hoff, J. H. Studies in Chemical Dynamics, Frederik Muller \& Co., Amsterdam, 1894.

[21] McC. Lewis, W. C. J. Chem. Soc., 1918, 113, 471-492.

[22] Hinshelwood, C. N. The Kinetics of Chemical Change, Oxford University Press, Oxford, 1940.

[23] Glasstone, S.; Laidler, K. J.; and Eyring, H. The theory of Rate Processes, McGraw-Hill, New York, 1941.

[24] Note that the two aforementioned theories have a temperature dependent preexponential factor, so that if this factor is assumed constant in computations the estimated activation energy may exhibit some small dependence as a computational artifact.

[25] Vyazovkin, S. New J. Chem., 2000, 24, 913.

[26] Hulett, J. R. Q. Rev. Chem. Soc., 1964, 18, 227.

[27] Vyazovkin, S. Thermochim. Acta, 397, 2003, 269.

[28] Brown, M. E.; Maciejewski, M.; Vyazovkin, S.; Nomen, R.; Sempere, J.; Burnham, A.; Opfermann, J. J.; Strey, R.; Anderson, H. L.; Kemmler, A.; Keuleers, R.; Janssens, J.; Desseyn, H.O.; Li, C. -R.; Tang, T.B.; Roduit, B.; Malek, J.; Mitsuhashi, T. Thermochim. Acta, 2000, 355, 125.

[29] Maciejewski, M. Thermochim. Acta 355, 2000, 145.

[30] Burnham, A. Thermochim. Acta 2000, 355, 165.

[31] Alexandre, M.; Dubois, P. Mater. Sci. Eng., R 2000, 28, 1. 
[32] Porter, D. E.; Metcalfe, E.; Thomas, M. J. K. Fire Mater. 2000, 24, 45.

[33] Noh, M.W.; Lee, S. C. Polym. Bull. 1999, 42, 619.

[34] Okamoto, M.; Morita, S.; Tafuchi, H.; Kim, Y. H.; Kotaka, T.; H. Tateyama, H. Polymer, 2000, $41,3887$.

[35] Wang, J.; Zhu, J.; Yao, Q.; Wilkie, C. A. Chem. Mater., 2002, 14, 3837.

[36] Zhu, J.; Wilkie, C. A. Polym. Int. 2000, 49, 1158.

[37] Gilman, J. W.; Jackson, C. L.; Morgan, A. B.; Harris, P.; Manias, E.; Giannelis, E. P.; Wuthenow, M.; Hilton, D.; Phillips S. H. Chem. Mater.,2000, 12, 1866.

[38] Morgan, A. B.; Harris, R. H.; Kashiwagi, T.; Chyall, L. J.; J. W. Gilman, J. W. Fire Mater., $2002,26,247$.

[39] ] Yoshioka, M.; Hancock, B. C.; Zografi, G. J. Pharm. Sci., 1994, 83, 1700.

[40] Andronis, V.; Zografi, G. J. Non-Cryst, Solids 2000, 271, 236.

[41] Hancock, B. C.; Shamblin, S. L.; Zografi, G. Pharm. Res., 1995, 12, 799.

[42] Johari, G. P.; Goldstein, M. J. Chem. Phys., 1970, 53, 2372.

[43] Vyazovkin, S.; Dranca, I. Pharm. Res., 2006, 23, 2158.

[44] Fan, X.; Xia, C.; Advincula, R. C. Colloids Surfaces: A, 2003, 219, 75.

[45] Vyazovkin, S.; Dranca, I.; Fan, X.; Advincula, R. Macromol. Rapid. Commun. 25, 2004, $25,498$.

[46] Vyazovkin, S.; Dranca, I. J. Phys. Chem. B, 2005, 109, 18637.

[47] Moynihan, C. T.; Lee, S.-K.; Tatsumisago, M.; Minami, T. Thermochim. Acta 1996, 280/281, 153.

[48] Donth, E. The Glass Transition: Relaxation Dynamics in Liquids and Disordered Materials; Springer: Berlin, 2001.

[49] Vyazovkin, S. J. Comput. Chem. 1997, 18, 393.

[50] Vyazovkin, S. J. Comput. Chem. 2001, 22, 178.

[51] Flynn, H.; Wall, L. A. J. Res. Nat. Bur.Stand.: A, 1966, 70, 487.

[52] Ozawa, T. Bull. Chem. Soc. Jpn., 1965, 38, 1881.

[53] Vyazovkin, S. Int. Rev. Phys. Chem. 2000, 19, 45.

[54] Hodge, I. M. J. Non-Cryst. Solids 1994, 169, 211.

[55] Gao, H.; Harmon, J. P. Thermochim. Acta 1996, 284, 85.

[56] McCrum, N. G.; Read, B. E.; Williams, G. Anelastic and Dielectrtic Effects in Polymeric Solids; Dover: New York, 1991.

[57] Boller, A.; Okazaki, I.; Wunderlich, B. Thermochim. Acta, 1996, 284, 1.

[58] Schawe, J. E. K. J. Polym. Sci. B 1998, 36, 2165.

[59] Simon, S. L.; Sobieski, J. W.; Plazek, D. J. Polymer 2001, 42, 2555.

[60] Donth, E. J. Polym. Sci. B 1996, 34, 2881.

[61] Van Krevelen, D. W. Properties of Polymers, $2^{\text {nd }}$ ed.; Elsevier: Amsterdam, 1976.

[62] Hempel, E.; Hempel, G.; Hensel, A.; Shick, C.; Donth, E. J. Phys. Chem. B 2000, 104, 2460.

[63] Karasz, F. E.; Bair, H. E.; O’Reilly, J. M. J. Phys. Chem.,1965, 69, 2657.

[64] ATHAS Database available at http://web.utk.edu/ athas/databank/intro.html.

[65] Abu-Hamdeh, N. H. Biosyst. Eng., 2003, 86, 97.

[66] Hancock, B. C.; Dalton, C. R.; Pikal, M. J.; Shamblin, S. L. Pharm. Res., 1998, 15, 762.

[67] Adams, G.; Gibbs, J. H. J. Chem. Phys., 1965, 43, 139. .

[68] Shamblin, S. L.; Tang, X.; Chang, L.; Hancock, B. C.; Pikal, M. J. J. Phys. Chem. B. 1999, 103, 4113.

[69] Busstin, O.; Descamps, M. J. Chem. Phys., 1999, 110, 10982.

[70] Wungttanagorn, R.; Schmidt, S. J. Thermochim. Acta 2001, 369, 95.

[71] Vyazovkin, S.; Dranca, I. J. Phys. Chem. B, 2004, 108, 11981.

[72] Carpentier, L.; Descamps, M. J. Phys. Chem. B, 2003, 107, 271.

[73] Faivre, A.; Niquet, G.; Maglione, M.; Fornazero, J.; Jal, J. F.; David, L. Eur. Phys. J. 1999, $10,277$.

[74] Kawai, K.; Hagiwara, T.; Takai, R.; T. Suzuki, T. Pharm Res. 2005, 22, 490.

[75] Noel, T. R.; Parker, R.; Ring, S. G. Carbohydr. Res. 2000, 329, 839.

[76] Fox, T. G.; Flory, P.J. J. Appl. Phys. 1950, 21, 581.

[77] McLoughlin, J. R.; Tobolsky, A. V. J. Coll. Sci. 1952, 7, 555.

[78] Orford, P. D.; Parker, R.; Ring, S. G. Carbohydr. Res. 1990, 196, 11.

[79] Illers, K. -H. Makromol. Chem., 1969, 127, 1.

[80] Chen, H. S, J. Appl. Phys. 1981, 52, 1868.

[81] Bershtein, V. A.; Egorov, V. M. Differential Scanning Calorimetry of Polymers, Ellis Horwood, New York, 1994.

[82] Boyer, R. F. Polymer, 1976, 17, 996.

[83] Gangasharan; Murthy, S. S. N. J. Phys. Chem. 1995, 99, 12349.

[84] Chan, R. K.; Pathmanathan, K.; Johari, G. P. J. Phys. Chem. 1986, 90, 6358.

[85] Kudilik, A.; Benkof, S.; Blochowitcz, T.; Tschirwitz, C.; Rössler, E. J. Mol. Str. 1999, 479, 201. 